\title{
The Matrix Effect on the Thermal Reactions of Spirooxazine in Polymer Matrices
}

\author{
Yasumitsu Munakata, Tetsuo Tsutsui, and Shogo Saito \\ Department of Materials Science and Technology, \\ Graduate School of Engineering Sciences, \\ Kyushu University, \\ Kasuga, Fukuoka 816, Japan
}

(Received February 5, 1990)

\begin{abstract}
The matrix effect on the thermal reactions of photochromic spirooxazine in polymers was investigated from the view points of reaction kinetics. Three matrix polymers, poly( $n$-butyl methacrylate), poly(isopropyl methacrylate), and poly(ethyl methacrylate) which possesed different $T_{\mathrm{g}}$ were used. Below $T_{\mathrm{g}}$ of plymer films, the thermal reactions did not proceed in a first-order kinetics and were found to be described with dispersive processes in glassy solids. The thermal reactions were influenced by molecular environments around the photochromic compounds. From the comparison of the rates of the thermal reactions in polymer matrices with those in solutions, the acceleration of the thermal reactions in solid states due to internal strains was found.
\end{abstract}

KEY WORDS Spirooxazine / Photochromism / Matrix Effect / Thermal Reaction / Internal Strains /

Reactions of photochromic compounds embedded in solid matrices are different from those in solutions. The kinetics of thermal reactions of photochromic molecules from metastable species to stable species do not proceed in a first-order kinetics in glassy matrices in particular. The departures from first-order kinetics behavior in polymer matrices have been observed in azobenzenes and indolinospirans, and in terms of the rates of the thermal reactions in polymer matrices, both cases that the rates in polymer matrices were slower and faster than those in solutions have been observed. ${ }^{1-5}$

The thermal reactions in polymer matrices are affected by the environment around photochromic molecules. Richert et al. showed the quantitative analysis of the departure from first-order kinetics of the thermal reaction in glassy matrices, assuming that each molecule in different molecular environment possessed different activation energy of thermal reaction.
They determined the average and the spread of the activation energies based on the assumption that the distribution of activation energy can be approximated by the Gaussian function. The fluctuation of the activation energies for thermal reactions derived from their analysis of thermal decay curves was large at temperatures below glass transition temperature $\left(T_{\mathrm{g}}\right)$ of matrix polymer and it approached zero above the $T_{\mathrm{g}}{ }^{6-8}$

We have analyzed quantitatively the effects of polymer matrices on the thermal reactions of photochromic molecules using the phenomenological procedure based on the KohlraushWilliams-Watts (KWW) equation, which is known to describe various types of non-linear relaxation processes in disordered solids. ${ }^{9}$ In that paper, we showed that, in glassy states, the molecular environment around photochromic probes was not uniform and the extent of non-uniformity was very wide. In addition, we found that the observed extent of the 
distribution depended on the method of photo-coloration. ${ }^{10,11}$ This meant that the dynamic aspect of molecular environment around photochromic probes should be taken into account.

The rates of the thermal reactions in polymer matrices have been assumed to be smaller than those in solutions, since thermal reactions in polymer matrices are constrained by the rigidity of polymer matrices. However, several cases that the rates of the thermal reactions in glassy solids are larger than those in solutions have been reported. ${ }^{12-14}$ Thus, both the extent of deviation from first-order kinetics and rates of thermal reactions is expected to be associated with the dynamic aspect of molecular environment, for example, segmental mobility of polymer matrices, the volume change accompanied by the structural changes of photochromic molecules, and inherent-rates of thermal reactions of photochromic molecules.

In this paper, we investigated the thermal reactions of spirooxazine in polymer matrices quantitatively. Our interests were on both the deviation from first-order kinetics and the rate of the thermal reactions.

\section{EXPERIMENTAL}

\section{Materials and Experimental Procedures}

As a photochromic probe, 1,3,3'-trimethylspiro (indoline-2', $3^{\prime}-(3 H)$ naphth $(2,1-\mathrm{b})(1,4)$ oxazine) (SO) shown in Figure 1, which was synthesized according to the literature, was used. ${ }^{15}$ On irradiation with UV light, SO changes to a colored species with an absorption maximum around $600 \mathrm{~nm}$, and a thermal-back reaction from a colored species (metastable) to a colorless species (stable) occurs in a time scale of a few seconds in a solution. Three matrix polymers, poly(n-butyl methacrylate) (PnBMA, $T_{\mathrm{g}}=289 \mathrm{~K}$ ), poly(isopropyl methacrylate) (PiPMA, $T_{\mathrm{g}}=354 \mathrm{~K}$ ) and poly(ethyl methacrylate) (PEMA, $T_{\mathrm{g}}=332 \mathrm{~K}$ ) were used. Three polymers possess similar chemical structures but their $T_{\mathrm{g}}$ values are markedly

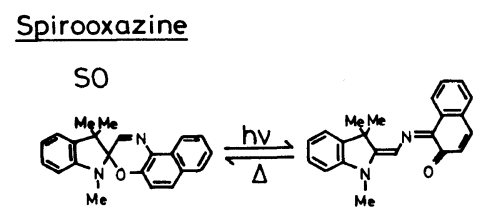

Polymer matrices

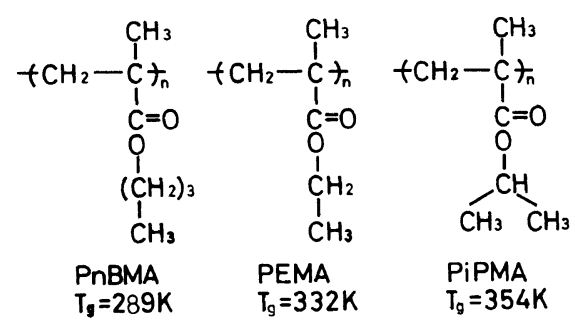

Figure 1. Photochromic probe, spirooxazine and three kinds of polymer matrices used.

different from each other. Those polymers were obtained by free radical polymerization in bulk and then were purified through reprecipitation. Polymer films of $50-60 \mu \mathrm{m}$ thickness which contained about $0.5 \mathrm{wt} \%$ photochromic probes were prepared by solution casting.

A sample film was set in an optical cryostat (Oxford DN-1754) and photo-coloration and thermal decoloration experiments were performed at temperatures around and below $T_{\mathrm{g}}$ of each film. For photo-coloration of SO, $308 \mathrm{~nm}$ laser pulses from a $\mathrm{XeCl}$ excimer laser (Lambda Physik EMG 50E) were used. The variation of concentration of colored species was observed by using a monochromatized monitor light $(600 \mathrm{~nm})$ from a halogen lamp and a photomultiplier. By used of a digital multimeter with a memory function (TakedaRiken TR-6861) and a transient converter (Riken-Denshi TCJ-2000), we collected time traces of the absorbance after the cessation of photoirradiation. Collected data were transfered to a personal computer (NEC PC-9801F) and analyzed.

\section{Analysis of Kinetics of the Thermal Back Reaction}

For the description of non-exponential decay 
of photo-colored species, we adopt one of the most general and simplest analytical formulation, which is based on no specified model of reaction kinetics.

Equation 1 describes a simple exponential decay of a colored species with a characteristic rate constant, $k$, where $C(t)$ represents the concentration of a colored species at time $t$. This equation means that the inherent reaction rate constant, $k$, is determined experimentally from $-\mathrm{d} \ln C(t) / \mathrm{d} t$ values at any observation time.

$$
-\mathrm{d} \ln C(t) / \mathrm{d} t=k
$$

If we assume an apparent reaction rate constant observed at time $t$ is dependent on observation time, eq 1 may be written as,

$$
-\mathrm{d} \ln C(t) / \mathrm{d} t=K(t)
$$

Integration of eq 2 gives,

$$
C(t) / C(0)=\exp \left[-\int_{0}^{t} K(t) \mathrm{d} t\right]
$$

One finds that $K(t)$ in eq 3 behaves as a kind of relaxation functions which describe various types of relaxation phenomena. Recent theoritical studies have shown that the relaxation functions which describe a variety of phenomena in disordered solids can be approximated by the stretched exponential function called Kohlraush-Williams-Watts (KWW) equation. ${ }^{16-19}$ Thus we express $K(t)$ with the KWW equation.

$$
K(t)=\kappa t^{\alpha-1} \quad(0<\alpha \leqq 1)
$$

When $\alpha$ takes unity, $K(t)=\kappa$. The parameter $\alpha$, therefore, represents the extent of deviation from a simple first-order reaction kinetics.

For simplicity, we define time-dependent reaction rate constant $k_{t}$ which is experimentally determined from the quantity, $-\mathrm{d} \ln C(t) /$ $\mathrm{d} t$,

$$
\log k_{t}=(\alpha-1) \log t+\log \kappa .
$$

When $\log k_{t} v s . \log t$ plots give straight lines, we obtain two parameters, $\alpha$ and $\kappa$. The quantity $\alpha$ mainly concerns with the deviation of reaction kinetics from the first-order reactions. On the other hand, $\kappa$ concerns with absolute reaction rates. Because the treatment of absolute reaction rate constants in non-first order reaction systems is extremely difficult, we do not dare to evaluate $\kappa$ values directly. Instead, we use $k_{t}$ values for the comparison of absolute reaction rates.

The thermal decoloration of colored species at fixed temperature in polymer matrices are influenced by both inherent reaction rates of colored species and the nature of matrix polymers. To extract the effect due to the nature of matrix polymers from overall reaction rate data, we nomalized the kinetics data in respect to inherent reaction rates which can be represented by the rates in solutions. In solutions, no environmental effect on thermal decoloration should be assumed, and fortunately the rates of SO in solutions are very weakly dependent on the polarity of solvents. Taking a reaction rate in solutions, $k_{s}$,

$$
\log \left(k_{t} / k_{s}\right)=(\alpha-1) \log t+\log \left(\kappa / k_{s}\right)
$$

Equation 6 was used for every plot at several fixed measuring temperatures. Note that both $k_{t}$ and $k_{s}$ have the dimension of $\mathrm{s}^{-1}$, but $\kappa$ and $k_{s}$ has a different dimension which depends on the $\alpha$ values.

\section{RESULTS AND DISCUSSION}

The thermal reaction of SO in $n$-heptane proceeded in first-order kinetics as shown in Figure $2 \mathrm{a}$. Figure $2 \mathrm{~b}$ shows the first-order kinetic plots of the thermal reaction of $\mathrm{SO}$ in PnBMA. The $T_{\mathrm{g}}$ value of the film was $289 \mathrm{~K}$. We observed the thermal reaction in wide temperature range below and above $T_{\mathrm{g}}$. The thermal reactions did not follow first-order kinetics except for the plot of data at $330 \mathrm{~K}$. Figure 3 shows the plots based on eq 5 . At every temperature, the plots could be approximated by straight lines. Straight-line approximations between $\log k_{t}$ and $\log t$ holded at 

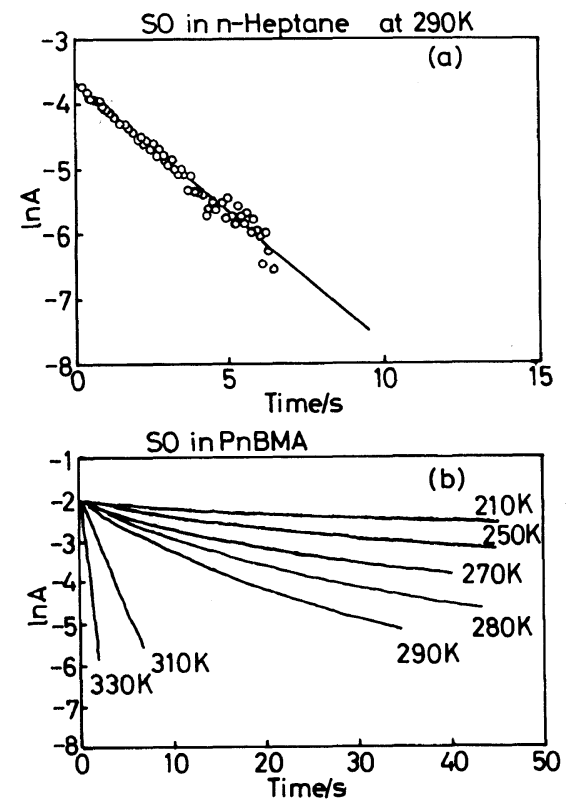

Figure 2. Fist-order kinetic plots of thermal reactions: (a) SO in $n$-heptane; (b) SO in PnBMA.

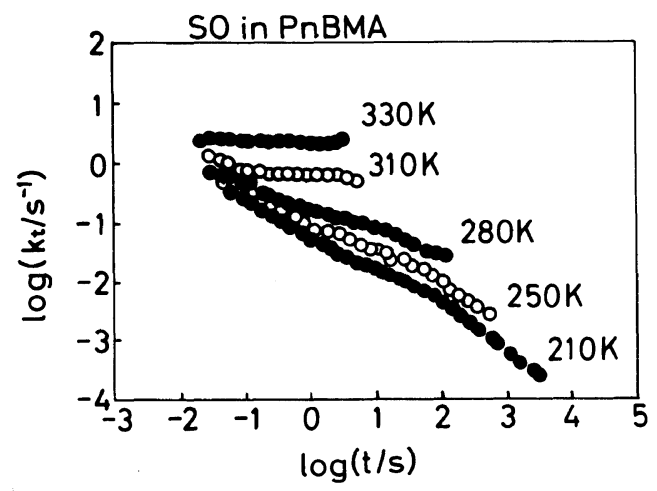

Figure 3. Analysis of the thermal reaction of $\mathrm{SO}$ in PnBMA by use of eq 5 .

wide range of time scale, exceeding as large as five decades. The parameter $\alpha$ was determined from the slopes of the lines.

Figure 4 shows the relation between the parameter $\alpha$ and temperature. The abscissa was reduced by the $T_{\mathrm{g}}$ of the film. Below the $T_{\mathrm{g}}$, the parameter $\alpha$ was very small and suggested the distribution of reaction rates was large. Around the $T_{\mathrm{g}}$, the $\alpha$ value rapidly increased

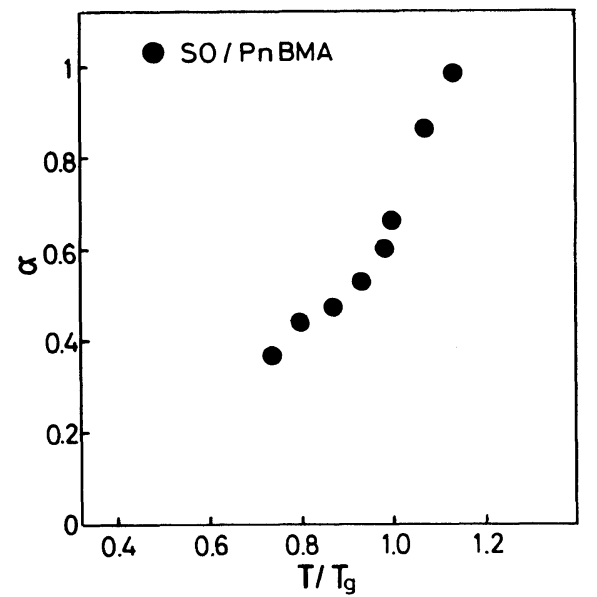

Figure 4. Parameter $\alpha$ plotted against $T / T_{\mathrm{g}}$ on the thermal reaction SO in PnBMA.

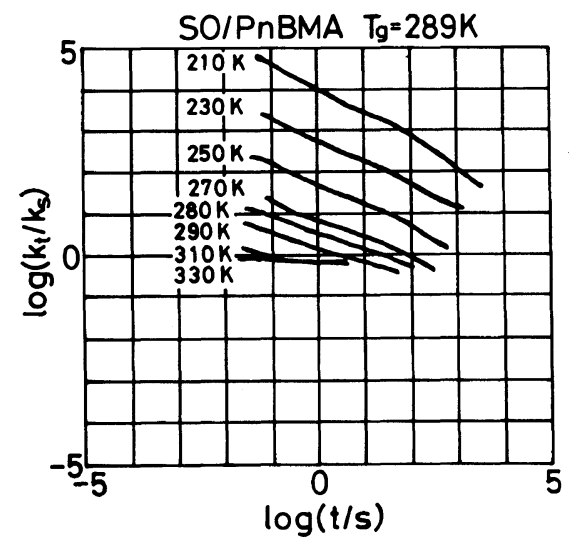

Figure 5. Analysis of the thermal reaction of SO in PnBMA by use of eq 6: $k_{s}$, rate constant in solution.

with increasing temperature, and above $T_{\mathrm{g}}$, the parameter $\alpha$ took a value close to unity. The thermal decay behavior was governed by the molecular environment of polymer matrix. Namely, below the $T_{\mathrm{g}}$, the thermal reaction is influenced by the microscopic inhomogeneity of molecular environments around photochromic probes. Segmental motions of matrix polymers work to smear the molecular environment around photochromic probes. Thus, the parameter $\alpha$ increases and progressively reaches to unity with increasing temperature at around and above $T_{\mathrm{g}}$. 
The comparison of the rates of thermal reactions in polymer matrices with those in solutions is possible, even though the reactions in polymer matrices do not obey the first-order kinetics. We compared the rates of the thermal reactions in polymer matrices with the rate of the thermal reactions in the solution ( $n$ heptane, concentration $\left.1 \times 10^{-3} \mathrm{M}\right)$. Figure 5 shows the plots of the thermal reactions of SO/PnBMA by use of eq 5 . The abscissa and ordinate reveal logarithmic time and logarithm of $k_{t} / k_{s}$, respectively. When the plot is on the horizontal zero line, the reaction is first-order and suffers no effect from environment. Above the $T_{\mathrm{g}}$, the plots locates close to horizontal zero line. Below the $T_{\mathrm{g}}$, however the curves located above zero line indicating that the reaction rates in polymer matrix became larger than those in a solution. Figure 6 shows the same analyses on the cases of SO/PEMA and SO/PiPMA. In the cases of SO/PEMA and SO/PiPMA, the behaviors below $T_{\mathrm{g}}$ were similar with that in SO/PnBMA.

To begin with, we investigated the possibility
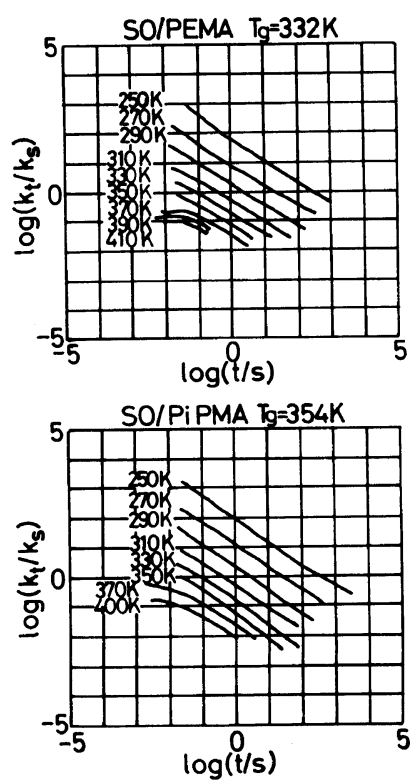

Figure 6. Analysis of the thermal reactions of SO in PEMA and PiPMA by use of eq 6: $k_{s}$, rate constant in solution. that the fast reaction is originated from some extrinsic matters. First, we examined transient heating due to UV pulses. When we assume that all the energy of UV pulses is converted to heat and no cooling effect due to thermal conduction is present, the estimated temperature increase is about $5 \mathrm{~K}$. Because not all the energy of UV pulses is absorbed by a sample film, and cooling effect is also present, actual temperature increase should be less than $5 \mathrm{~K}$. Thus the presence of $k_{t} / k_{s}>0$ at the initial stage of the thermal reaction is not associated with transient heating of sample films. Second, we considered the photo-decoloration with monitor light. The rate of decoloration did not depend on the intensity of monitor light. Therefore, we confirmed that the observed decolorations were true thermal reactions at isothermal conditions.

Thermal reactions in rigid polymer matrices have been recognized to be slower than those in mobile solutions due to steric hindrance around photochromic probes. Our results show that the reaction rates in glassy matrices can be larger than those in a solution, in the case that the colored species produced by photoirradiation in rigid glassy matrices possess some kinds of internal strains.

Several reports are available that deal with anomalous fast thermal back reactions of azobenzene derivatives in glassy polymer matrices. ${ }^{12-14}$ Two different interpretations of this phenomenon have been proposed. Morawetz and Paik studied the thermal cis-trans isomerization of copolymers of methacrylate and styrene with azobenzene units in their side chains. They observed an anomalous fast reaction of cis isomers in glassy states and attributed the origin of the fast isomerization to the presence of cis isomers in strained conformation. Eisenbach studied the isomerization process of copolymer of several acrylates and methacrylates attached by 4-aminoazobenzene derivatives. He also found an anomalous fast isomerization process and attributed it to high local segmental mobility 
around the azocompounds.

We assumed that the anomalous fast reaction of SO in rigid polymer matrices should be attributed to the presence of colored species of SO in strained conformation which failed to push rigid environment around itself. Because colored species of SO have a conjugated system, the absorption spectrum of the colored species in strained conformation may be shifted to longer wavelength. We observed the absorption spectra of colored species of SO in PnBMA at $430 \mathrm{~K}$ and $80 \mathrm{~K}$. We could find no difference between the spectra at $430 \mathrm{~K}$ and $80 \mathrm{~K}$. Probably the wavelength shift due to strained conformation is too small to be detected with conventional spectroscopic methods.

The study of photochromism of SO in glassy matrices at around $10 \mathrm{~K}$ is in progress. We expect the molecular origin of the anomalous fast back reaction in some glassy matrices will become clear through this study.

\section{CONCLUSION}

In the present work, we have investigated the matrix effect for the thermal reactions of spirooxazine in some methacrylate polymers. We applied KWW type decay to the thermal reactions in polymer matrices. In every glassy polymer matrix we used, the thermal reactions showed large departure from the first-order kinetics and the parameters which characterized the distribution of molecular environments around photochromic molecules were determined by use of KWW decay function. Above the $T_{\mathrm{g}}$ of polymers, the thermal reactions proceed in a first-order kinetics, because homogeneity of molecular environments was preserved due to segmental motions of matrix polymers. Below the $T_{\mathrm{g}}$, on the other hand, large distribution of molecular environment was found.

We compared the rates of thermal reactions in polymer matrices with those in solutions. The rates of the thermal reactions in glassy states were accelerated. We assumed that the effect was originated from the conformational strains of distorted colored species.

\section{REFERENCES}

1. G. Smets, Adv. Polym. Sci., 50, 17 (1983).

2. J. Guillet, "Polymer Photophysics and Photochemistry," Cambridge University Press, Cambridge, 1985, p 95.

3. C. D. Eisenbach, Photog. Sci. Eng., 23, 183 (1979).

4. M. Kryszewski and B. Nadolski, J. Chem. Soc., Faraday Trans. 2, 76, 351 (1983).

5. K. Horie and I. Mita, Adv. Polym. Sci., 88, 77 (1989).

6. R. Richert and H. Bassler, Chem. Phys. Lett., 116, 302 (1985).

7. R. Richert, Chem. Phys. Lett., 118, 534 (1985).

8. R. Richert, A. Elschner, and H. Bassler, Z. Phys. Chem., 149, 63 (1986).

9. G. Williams and D. C. Watts, Trans. Faraday Soc., 66, 80 (1970).

10. T. Tsutsui, A. Hatakeyama, S. Saito, and M. Irie, Chem. Phys. Lett., 132, 563 (1986).

11. Y. Munakata, T. Tsutsui, and S. Saito, Rep. Prog. Polym. Phys. Jpn., 30, 569 (1987).

12. C. S. Paik and H. Morawetz, Macromolecules, 5, 171 (1972).

13. C. D. Eisenbach, Makromol. Chem., 179, 2489 (1978).

14. I. Mita, K. Horie, and K. Hirao, Macromolecules, 22, 558 (1989).

15. N. K. C. Chu, Can J. Chem., 61, 300 (1983).

16. A. Bulumen, J. Klafter, and G. Zumofen, Phys. Rev., B27, 3429 (1983).

17. M. Shlesinger and E. W. Montroll, Proc. Natl. Acad. Sci. U.S.A., 81, 1280 (1984).

18. J. Klafter and M. Shlesinger, Proc. Natl. Acad. Sci. U.S.A., 83, 848 (1986).

19. J. Klafter and A. Bulumen, Chem. Phys. Lett., 119, 377 (1985). 